\title{
A novel mathematical modelling for simulating the spread of heavy metals in solid waste landfills
}

\author{
Ngoc Ha Hoang1, Anh My Chu' ${ }^{2}$ Kim Thai Thi Nguyen ${ }^{1}$, Chi Hieu Le ${ }^{3^{\dagger}}$ \\ ${ }^{1}$ Department of Environment Engineering, National University of Civil Engineering, Hanoi, Vietnam \\ ${ }^{2}$ Institute of Simulation Technology, Le Quy Don Technical University, Hanoi, Vietnam \\ ${ }^{3}$ Faculty of Engineering and Science, University of Greenwich, Kent ME4 4TB, United Kingdom
}

\begin{abstract}
Municipal solid waste (MSW) landfills create the environmental pollutions due to problems related to toxins, leachate, and greenhouse gas, which are the growing environmental concerns, especially in developing countries. This paper presents a novel mathematical modelling for simulating the spread of heavy metals in soil layers in the MSW landfills. A new mathematical modelling procedure is studied for deriving the governing equation which describes the time varying concentration of pollutants in the leachate in a 3-dimensional (3D) space of soil layers. A Finite Element Method (FEM) based algorithm is then constructed to solve numerically the governing equation over time in 3D space for effectively analyzing the variation of pollutants concentrations. Finally, the applicability and advantages of the proposed novel method are demonstrated through the case study with soil samples collected from the actual MSW landfill. The successfully developed algorithm can be applied to model and predict the spread of different heavy metals in the MSW landfill sites; and to develop effective solutions and policies for solid waste classification and management, in order to minimise the negative impacts from the potential risk of the toxic heavy metal pollutions, soil contaminations and groundwater pollutions in the areas nearby the MSW landfill.
\end{abstract}

Keywords: Analytical Modeling, Finite Element Method, Heavy Metal Pollution, Leachate Immigration, Municipal Solid Waste

\section{Introduction}

The inefficient management and uncontrollable disposal of the municipal solid waste (MSW) in big cities, especially in developing countries, causes serious problems to the environmental contamination. Effective controls of the MSW landfills have become one of the biggest challenges to the national and local authorities. The key constraints for management of the MSW landfills include the limitation of land space and landfill sites for dumping solid wastes, as well as the overload and shortage of skilled manpower. The unscientific control and processing of the MSW landfills often lead to several critical issues such as the foul odor generation and the leachate immigration into the water streams that affect seriously the public health and society [1]. Especially, the untreated leachate from the MSW landfills causes potential risks to the quality of underground water and eventually to the health of residents living near the landfill sites.

In developing countries such as Vietnam, most of the MSW landfills are not segregated at source and they are daily collected from residential areas to dispose directly at landfill sites. Giang et al. [2] showed that about $70 \%$ of the MSW landfills in Vietnam are improperly implemented, overloaded or operated in an inefficient and insanitary manner. The migration of the leachate from the landfills to soil layers leads to degradation of soils, air pollution and contamination of groundwater sources in the areas nearby the landfills. It is more important that the leachate from the MSW landfills contains a wide spectrum of hazardous environmental contaminants [3]. When the leachate streams infiltrate through the bottom soil layers, the physico-chemical properties of the groundwater sources are affected, and the groundwater contamination occurs. Sabour and Amiri [4] indicated that even a small amount of leachate infiltration into groundwater or surface water, a large volume of water resources can be polluted; moreover, among the different pollutants in the leachate immigration, heavy metals are of significant concerns to the pollution of groundwater source. It was concluded that the heavy metals trapped in the
This is an Open Access article distributed under the terms of the Creative Commons Attribution Non-Commercial License (http://creativecommons.org/licenses/by-nc/3.0/) which permits unrestricted non-commercial use, distribution, and reproduction in any medium, provided the original work is properly cited.

Copyright (C) 2022 Korean Society of Environmental Engineers
Received January 01, 2021 Accepted April 24, 2021

${ }^{\dagger}$ Corresponding author

E-mail: C.H.Le@gre.ac.uk

Tel: +44 (0)1634 883050

ORCID: 0000-0002-5168-2297 
soil beneath dumpsites result in the long-term contamination of the underlying soil [5]; and heavy metals were identified as the dominant pollutants and major contributing factors for the leachate pollution potential [6]. Gao et al. [7] showed that the main environmental impacts of the leachate pollution are the heavy metal ions and elements which exist in the leachate passing through layers of soil and reaching groundwater. The environmental contamination by heavy metals should be carefully taken into account in management of solid wastes, since the existence of the heavy metal ions and elements in the groundwater body affects directly on resident communities, plants, vegetables and animals using such the contaminated groundwater [8].

There have been a number of research works focusing on the pollution or contaminant transport mechanisms occurring in the landfills and the related effects on the groundwater contamination, with the use of mathematical modeling and simulations to study the spread of contaminants in the solid waste landfills. Sharma and Lewis [9] studied a mathematical model of the leachate immigration that includes the advection and dispersion of a pollutant contaminant. Varank et al. [10] investigated the migration of the contaminants in the landfill leachate through alternative composite liners. Benson et al. [11] took into account issues related to the field hydraulic conductivity of pollutant different types of clay liners, when studying the leachate immigration from a landfill site. The desiccation of a geosynthetic clay liner was also evaluated by Hoor and Kerry Rowe [12]. The bacterium in liners of a landfill was analyzed by Tang et al. [13]. Reddy et al. [14] examined the impact of a vertical well configuration on the leachate distribution in the MSW landfill.

Along with the aforementioned studies, there have also been a number of studies focusing on the formulation of the leachate immigration models and the numerical techniques to simulate the transportation of contaminants from the leachate into the soil layers [7, 14-27]. Recently, some numerical techniques were developed for solving the governing equation and simulation of the contaminant transport in the soil space [21, 25, 28, 29]; and the Finite difference (FD) and finite volume (FV) approaches were also used $[8,23,24,30]$. It can be seen that though there has been a number of studies focusing on the mathematical modelling of the leachate immigration in soil medium [15-17, 20, 22-29, 31, 32], a little attention has been paid to develop a comprehensive and compact mathematical modelling procedure by using the effective mathematical representation and expressions related to the gradient vector field. In several documented works, the governing equation was simplified by eliminating the advection terms or using the linearization technique for obtaining analytical solutions in the ideal conditions [15-18, $22,33,34]$; and the partial derivative equation is transformed and represented in one or two dimensions only [17, 19, 27, 31, 32, 35]. There also have been investigations focusing on the absolute permeability of water in a porous structure [36], and heavy metal contaminants that contribute to environmental pollution and raw agricultural waste [37, 38]. The publication [36] designed a Bayesian framework to qualify the permeability of water in a porous media by taking into account the geometry and parameters of the pore-throat network. The studies [37, 38] evaluated the treatment process of waste water both in the presence and absence of heavy metals.

Thus, it is necessary to investigate the untreated leachate immi- gration from MSW landfills into the environment system, to compute and predict reliably the pollution or contaminant transport mechanisms in soil layers, as well as to assess the related effects on the groundwater contamination, leading to the effective solutions for solid waste management and reduction of potential risks of soil contamination and groundwater pollution, especially for the cases in which the solid wastes are disposed directly to the landfills without any classification, and for the cases of uncontrollable MSW landfill sites. In order to predict reliably and effectively the pollutant contaminant transport in soil layers, it is necessary to develop a comprehensive mathematical modelling of the pollutant concentration in the leachate in 3D layers of soil, and to construct an efficient simulation algorithm.

In this study, a general approach which combines a mathematical modelling method and Finite Element Method (FEM) simulation is proposed to investigate the concentration of pollutants in the leachate passing through saturated soil layers. A case study of modelling and computation of the spread of the arsenic pollutant due to the leachate immigration into the soil layers and groundwater at the actual municipal solid waste landfill site was implemented to validate the proposed novel mathematical modeling and FEM-based algorithms, in which the initial value of arsenic concentrations was calculated by using the data from the experiments which are analyzed from the soil samples collected from the actual municipal solid waste landfill. The newly introduced method for modelling the spread of the pollutant concentration in the leachate immigration in the 3D space of soil layers, and the new algorithm for simulating the pollutant transport in the 3D space are the main contributions of this study.

The rest of the paper is organized as follows. Section 2 presents materials and methods, with the focus on the problem formulation and mathematical modelling of leachate migration from a landfill, as well as the FEM-based algorithm for solving the defined problem. Section 3 presents the results and discussions about the proposed numerical algorithm applied for solving the defined problem of the leachate migration from a landfill, with the specific case study. Finally, the conclusions are summarised and presented in Section 4.

\section{Materials and Methods}

Fig. 1 presents the flowchart, as a methodology for implementation of a study, with the related research tools and methods.

First of all, the study problem as well as requirements for mathematical modelling and simulations should be well-formulated and defined. A new mathematical modelling procedure is studied for deriving the governing equation which describes the time varying concentration of pollutants in the leachate in a 3-dimensional (3D) space of soil layers, with the use of the gradient vector field approach, gradient vector operators, and partial different equations. Then, FEM-based simulation algorithms are developed, to solve numerically the governing equation over time in 3D space for effectively analysing the variation of pollutants concentrations. Finally, the proposed mathematical modelling and the FEM-based algorithm are validated, analysed and discussed through the case study with soil samples collected from the actual MSW landfill, in comparison with the findings from the related studies. 


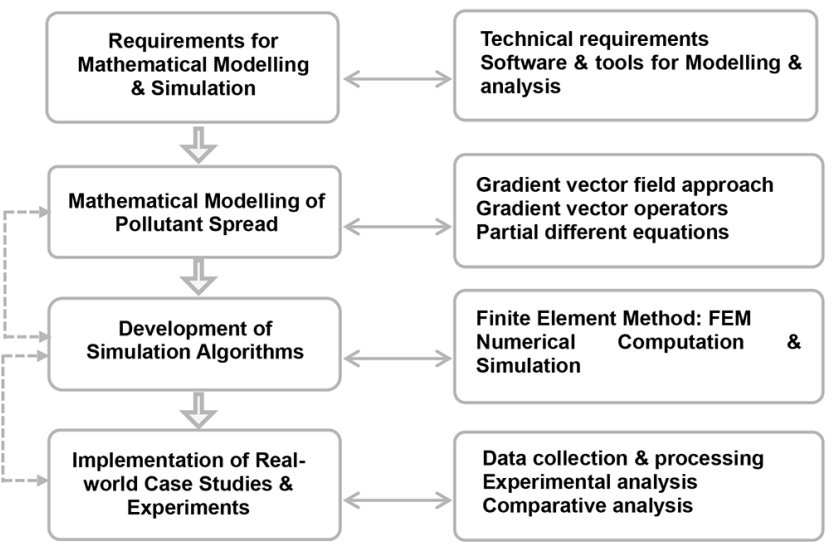

Fig. 1. Flowchart for implementation of a study.

\subsection{Data Collection and Analysis}

Fig. 2 presents the location of the Kieu Ky landfill in Hanoi, Vietnam, with the following key data and information presented in Table 1. The positions of the drilling holes for collection of soil samples for the experimental analysis are presented in Fig. 3; and the arsenic content in soil samples are analyzed via the use of the inductively coupled plasma mass spectrometry (ICP-MS) and high precision atomic emission spectrometry (AES).

As mentioned above, the problem formulation and new mathematical modelling of leachate migration from the MSW landfills are implemented and investigated, to derive the governing equation which describes the time varying concentration of pollutants in the leachate in a 3D space of soil layers, using the mathematical concepts related the gradient vector field. The FEM-based algorithm is then constructed to solve numerically the governing equation so that the variation of concentration of pollutants in the leachate can be analysed effectively in the 3D space overtime. Finally, the proposed mathematical modelling and the FEM-based algorithm are validated, analysed and discussed through the case study with soil samples collected from the actual MSW landfill, in comparison with the findings from the related studies. The soil samples were collected at the actual landfill: the Kieu Ky landfill in Hanoi, Vietnam as shown in Fig. 2 and Fig. 3, to determine the arsenic concentration in the nearby areas of the Kieu Ky landfill, which is used as the initial value to implement the proposed mathematical algorithm.

Table 1. Data and Information about the Kieu Ky Landfill Site in Hanoi, Vietnam

Total area (ha)

Waste source

Capacity (ton/d)

Waste disposal method

Height of the cell

Average depth of waste dump (m)

Hydrology
13

Municipal solid waste

170-180 ton/d

Sanitary landfill

From 5 to $12 \mathrm{~m}$

$4.5 \mathrm{~m}$

The underground water level is about $2.0 \mathrm{~m}$ from the ground.

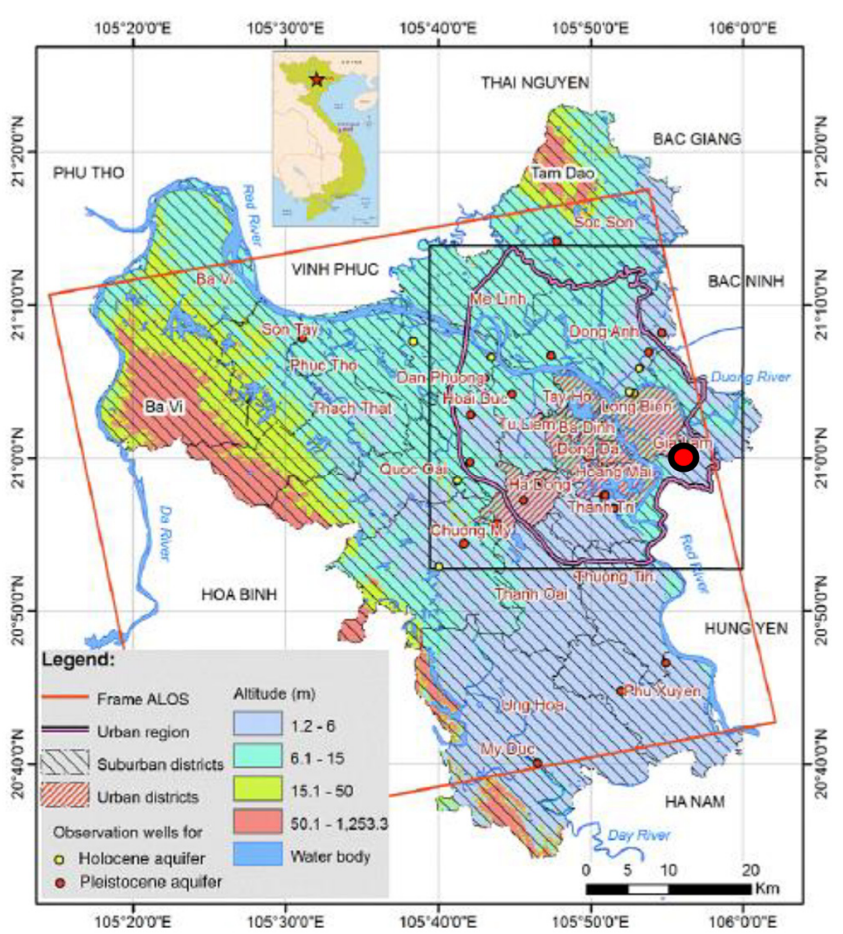

Fig. 2. Location of the Kieu Ky landfill: Kieu Ky Village, Gia Lam District, Hanoi, Vietnam.

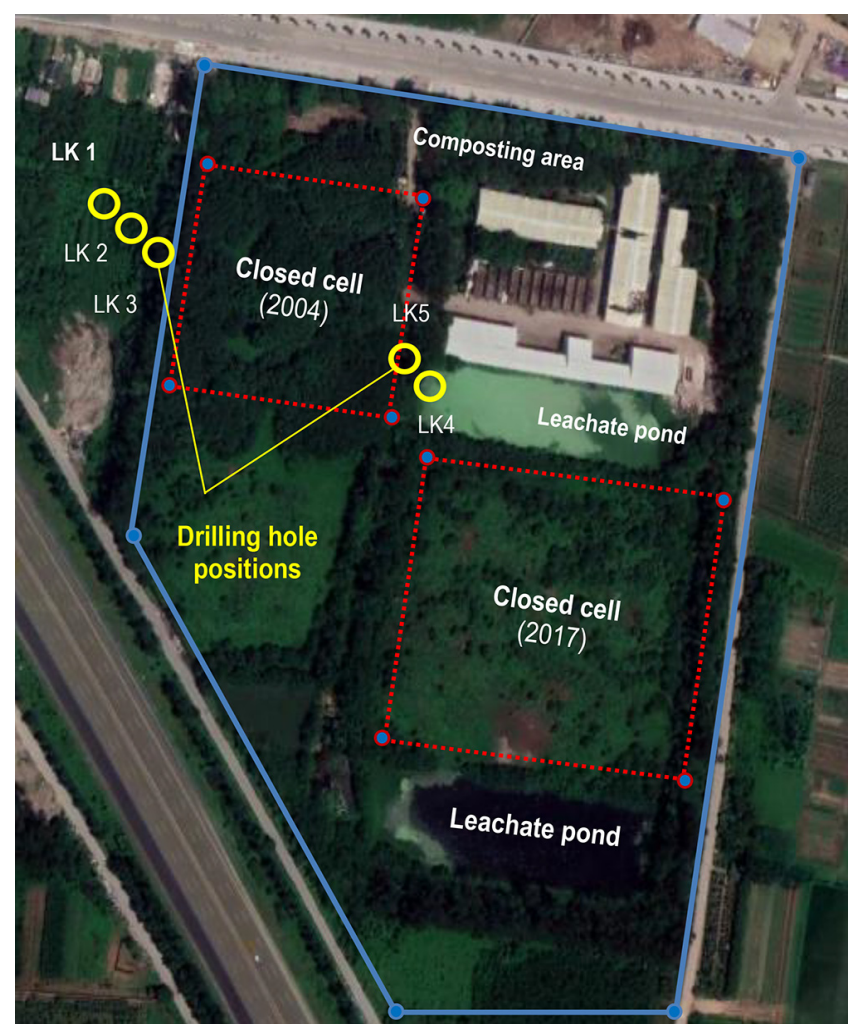

Fig. 3. The key information and data about the Kieu Ky landfill in Hanoi, Vietnam, and the drilling hole positions for collection of soil samples for the experimental analysis. 
Table 2. Collected Soil Samples at the Kieu Ky Landfill in Hanoi, Vietnam for the Experimental Analysis of Arsenic Concentrations

\begin{tabular}{|c|c|c|c|}
\hline No. & Depth of the drilling hole (m) & Characteristics of collected soil samples & Selected for analysis in Lab \\
\hline \multicolumn{4}{|c|}{ DRILL HOLE - LK4 } \\
\hline 1 & $0-0.3$ & Porous black-brown clay with roots and rubbish & \\
\hline 2 & $0.3-0.6$ & Porous black-brown clay with roots and rubbish & * \\
\hline 3 & $0.6-0.9$ & Porous black-brown clay with roots and rubbish & \\
\hline 4 & $0.9-1.2$ & Porous black-brown clay with roots and rubbish & * \\
\hline 5 & $1.2-1.5$ & Porous black-brown clay with roots and rubbish & \\
\hline 6 & $1.5-1.8$ & Black brown, smooth, sticky and fishy clay & * \\
\hline 7 & $1.8-2.1$ & Black brown, smooth, sticky and fishy clay & \\
\hline 8 & $2.1-2.4$ & Dark brown, clay mixed and gritty & * \\
\hline 9 & $2.4-2.7$ & Dark brown, clay mixed and gritty & \\
\hline 10 & $2.7-3.0$ & Sticky and fishy black brown clay & * \\
\hline 11 & $3.0-3.3$ & Black brown clay, sticky, fishy, smooth & \\
\hline 12 & $3.3-3.6$ & Black brown clay (dark) sticky & * \\
\hline 13 & $3.6-3.9$ & Black brown clay (dark) sticky & \\
\hline 14 & $3.9-4.2$ & Black powder clay mixed with fine sand & * \\
\hline \multicolumn{4}{|c|}{ DRILL HOLE - LK5 } \\
\hline 1 & $0-0.3$ & Black brown, loose, powder clay mixed with rubbish & \\
\hline 2 & $0.3-0.6$ & Black brown, loose, powder clay mixed with rubbish & * \\
\hline 3 & $0.6-0.9$ & Yellow clay, sticky & \\
\hline 4 & $0.9-1.2$ & Yellow clay, sticky & * \\
\hline 5 & $1.2-1.5$ & Yellow clay, sticky, mixed with bricks & \\
\hline 6 & $1.5-1.8$ & Mixed sand & * \\
\hline 7 & $1.8-2.1$ & Mixed sand & \\
\hline 8 & $2.1-2.4$ & Mixed sand, black brown, smooth and flexible & * \\
\hline 9 & $2.4-2.7$ & Mixed sand, black brown, smooth and flexible & \\
\hline 10 & $2.7-3.0$ & Mixed sand, black brown & * \\
\hline 11 & $3.0-3.3$ & Mixed sand, black brown & \\
\hline 12 & $3.3-3.6$ & Greenish gray clay, smooth and flexible & * \\
\hline 13 & $3.6-3.9$ & Greenish gray clay, smooth and flexible & \\
\hline 14 & $3.9-4.2$ & Dark green clay mixed with fine grained sand & * \\
\hline 15 & $4.2-4.5$ & Dark green clay mixed with fine grained sand & \\
\hline 16 & $4.5-4.8$ & Powder clay, fine grained sand, black brown & * \\
\hline 17 & $4.8-5.2$ & Powder clay, fine grained sand, black brown & \\
\hline 18 & $5.2-5.5$ & Powder clay, fine grained sand, black brown & * \\
\hline
\end{tabular}

Speficicatlly, for the case study of investigating the arsenic spread at the Kieu Ky landfill, 2 main steps are implemented as follows:

Step 1: Determining the arsenic concentration in the nearby areas of the Kieu Ky landfill by the empirical research based on the statistical methods.

Step 2: The result in Step 1 is used as the initial value to implement the proposed mathematical algorithm to study the arsenic propagation along the leachate in the ground.

The soil samples for the experimental analysis in the laboratory are collected from the drilling holes at locations LK1, LK2, LK3,
LK4 and LK5 in the Kieu Ky landfill as shown in Fig. 3. Maximum depth of the drilling hole is 6 meters. The soil samples were collected about 300 grams at each 0.3 meter of depth. The depth of the drilling hole and characteristics soil sample are presented in Table 2. Note that the data collected with the drilling holes LK1, LK2 and LK3 are used for reference purpose, since these holes are located outside the borderline (Blue line) of the investigation area. Table 2 presents the data of soil samples collected from the holes LK4 and LK5. The soil samples were kept in the tightly packed plastic bags and then analyzed in the laboratory for the arsenic content via the use of ICP-MS and AES. 


\subsection{Mathematical Modelling of Pollutant Transport in the Leachate Immigration from a Landfill}

Generally, the contaminant transport mechanisms in an unsaturated and porous soil environment consist of three main categories: advection through the leachate flow into the soil media, dispersion in moist soils, and other physicochemical interactions. Corresponding to these simultaneous transport mechanisms, three mathematical expressions must be written, to derive a unified governing equation for the transport.

To formulate the model of the contaminant transport in the leachate flow, some assumptions are given as follows. It is assumed that the soil layer is considered as a homogeneous medium in which the dispersion process is isotropic. All water particles move at the same velocity through the porous medium. It means that the microscopic variations in flow velocity is neglected. In addition, the contaminant concentration in the leachate flow is sufficiently small, so that the dispersion coefficient is independent of concentration, and it is approximated by a constant. Also, no chemical reaction is assumed to occur between the solid and the liquid phases. It means that within the leachate flow no loss or addition of matter can take place.

From a MSW landfill on the surface, at the same time, the leachate flow that contains heavy metal contaminants spreads in the vertical direction, down to the depth of the soil layers, and transport to the surrounding areas. The concentration of contaminants in the flow changes along all directions of the layers over time: $\mathrm{X}, \mathrm{Y}$ and Z (Fig. 4).

Let's denote $C(x, y, z, t)$ as a scalar function of concentration $(\mathrm{mg} / \mathrm{kg})$ of a pollutant in the leachate. The function $C(x, y, z, t)$ is represented in a Cartesian coordinate system Oxyz and changes over time $t$. As usual, the origin of the coordinate system $O x y z$ places on the ground surface of soil media, the axis $\mathrm{Oz}$ points vertically downward the depth of the soil layers. The concentration function $C(x, y, z, t)$ depends on four variables $x, y, z$ and $t$.

The advection mechanism of a substance (pollutant) refers to the transport of the pollutant itself with the mean leachate flow. Hence, at any point $(x, y, z)$ in the soil space, the change of the pollutant concentration $C(x, y, z, t)$ with velocities $\boldsymbol{v}=\left[\begin{array}{lll}u & v & w\end{array}\right]^{T}$ $[\mathrm{m} / \mathrm{s}]$ in three directions $\mathrm{x}, \mathrm{y}$ and $\mathrm{z}$, respectively can be represented effectively with Eq. (1), by using the concept of the concentration

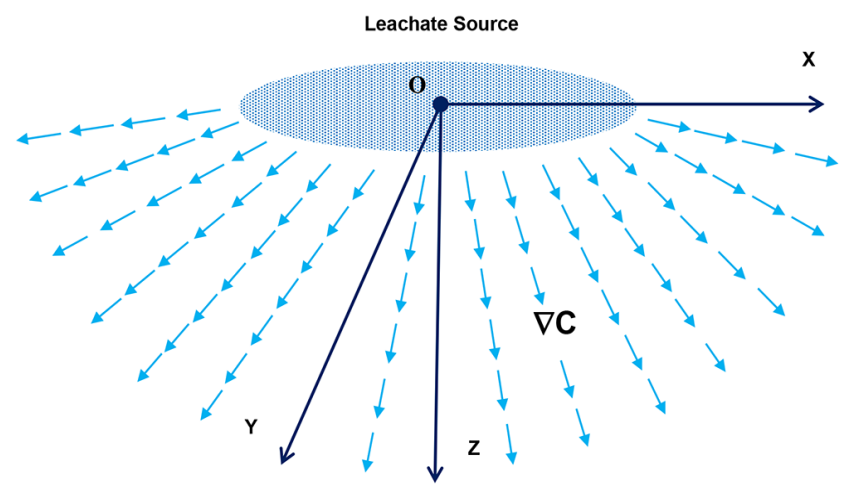

Fig. 4. Gradient field of the concentration function $C(x, y, z, t)$ in the coordinate system Oxyz. gradient vector $\nabla C(x, y, z, t)$ in the coordinate system $O x y z$ as demonstrated in Fig.4.

$$
\frac{\partial C}{\partial t}=-\nabla C \cdot \boldsymbol{v}
$$

where

$$
\nabla C=\left[\begin{array}{l}
\frac{\partial C}{\partial x} \\
\frac{\partial C}{\partial y} \\
\frac{\partial C}{\partial z}
\end{array}\right]
$$

When taking into account the dispersion of the pollutant concentration, the gradient vector $\nabla C$ and other relevant mathematical operators can be used as well to formulate the dispersion process model of a pollutant dispersion in the leachate.

It is assumed that when the concentration $C(x, y, z, t)$ of a pollutant at a given point $(x, y, z)$ is low somewhere compared to the surrounding points, physically the substance diffuses into the point $(x, y, z)$ from the surroundings, so the value of the concentration $C(x, y, z, t)$ is increased. Conversely, if the concentration $C(x, y, z, t)$ is high as compared with the surroundings, then the substance moves away and the concentration of the pollutant at the point $(x, y, z)$ is decreased. Finally, the net dispersion is proportional to the second derivative of the concentration function if the dispersion coefficient $\boldsymbol{D}=\left[\begin{array}{lll}D_{x} & D_{y} & D_{z}\end{array}\right]^{T}$ is a constant vector.

To this end, Eq. (1) becomes

$$
\frac{\partial C}{\partial t}=-\nabla C \cdot v+(\nabla \cdot)(D \odot \nabla C)
$$

It is noted that, in the second component of Eq. $(1),(\nabla \cdot)(\boldsymbol{D} \odot \nabla C)$, which represents the pollutant transport by dispersion, the notation $(\nabla \cdot)$ represents the divergence of the gradient field $\nabla C$, and the operator $\odot$ is the element-wise product (the Hadamard product) of two vectors.

If we take the third contribution $R$ which represents the change of the concentration $C(x, y, z, t)$ in the soil media space due to other chemical reactions and physicochemical interactions, the governing equation Eq. (2) can be rewritten in a generic form as follows:

$$
\frac{\partial C}{\partial t}=-\nabla C \cdot v+(\nabla \cdot)(D \odot \nabla C)-R
$$

As assumed earlier, the dispersion coefficient is independent of concentration, and it is approximated by a constant. Also, no physico-chemical reaction is assumed to occur between the solid and the liquid phases. Therefore, in this study, it is assumed that change of arsenic concentration due to physico-chemical effects is neglected. It means that the physicochemical reactions of the heavy metal contaminants in the leachate flow are not noticeable, and within the leachate flow no loss or addition of matter can take place. Hence, $R \simeq 0$. In other words, expressing Eq. (3) in 
terms of the partial derivatives of the concentration function $C(x, y, z, t)$ yields

$$
\begin{gathered}
\frac{\partial C}{\partial t}+u \frac{\partial C}{\partial x}+w \frac{\partial C}{\partial y}+v \frac{\partial C}{\partial z} \\
=\frac{\partial}{\partial x}\left(D_{x} \frac{\partial C}{\partial x}\right)+\frac{\partial}{\partial y}\left(D_{y} \frac{\partial C}{\partial y}\right)+\frac{\partial}{\partial z}\left(D_{z} \frac{\partial C}{\partial z}\right)
\end{gathered}
$$

Finally, the time variation of concentration of a pollutant when the leachate immigrates into the 3D space of soil media has successfully formulated with either Eq. (3) or its equivalent expression Eq. (4). These formulations play an important role when analyzing the time evolution of the concentration of a heavy metal in the leachate at any point in the soil media or to simulate and predict the spread of a heavy metal pollutant from a landfill site to the surrounding areas.

\subsection{Finite Element Algorithm to Solve Numerically the Governing Equation over Time in 3D Space for Effective Analysis of Variation of Pollutants Concentrations}

The pollutant concentration $C(x, y, z, t)$ simultaneously depends on 4 variables: $x, y, z$ and $t$. Mathematically, the variation of $C(x, y, z, t)$ over time $\mathrm{t}$ and $\mathrm{x}, \mathrm{y}, \mathrm{z}$ is explicitly described by the specific derivative relations as showed in Eq. (4). Moreover, the variation of $C(x, y, z, t)$ depends on 6 parameters as $\mathrm{u}, \mathrm{w}, \mathrm{v}, \mathrm{Dx}$, Dy and Dz. In practice, these parameters can be changed in soil domain even in time. Due to such the spatial and time-dependent complexity of the mathematical model, in this study, it is proposed that a FEM technique to solve the governing equation over time $t$ in $3 \mathrm{D}$ space $\mathrm{x}, \mathrm{y}, \mathrm{z}$ with an assumption that the permeability and dispersion of the pollutant concentration are similar in 2 horizontal directions $\mathrm{x}$ and $\mathrm{y}$. To discrete the equation with respect to a 3D space of finite elements, the finite difference method is used for both time variable and space variables $\mathrm{x}$ and z. This method can also be called as the finite element method with evenly spaced grid nodes at the distance $\Delta x$ and $\Delta z$. Accordingly, in a time that is small enough $\Delta t$, at a distance $\Delta x$ and $\Delta z$ small enough, the governing equation Eq. (4) at time $\mathrm{t}=\mathrm{n}$ is discrete as follows:

$$
\begin{gathered}
\frac{C_{i, j}^{n+1}-C_{i, j}^{n-1}}{2 \Delta t}+\left(\frac{u_{i+1, j}-u_{i-1, j}}{2 \Delta x}\right) C_{i, j}^{n}+\frac{u}{2 \Delta x} C_{i+1, j}^{n}- \\
\frac{u}{2 \Delta x} C_{i-1, j}^{n}+\frac{v_{i, j+1}-v_{i, j-1}}{2 \Delta z} C_{i, j}^{n}+\frac{v}{2 \Delta z} C_{i, j+1}^{n}-\frac{v}{2 \Delta z} C_{i, j-1}^{n}= \\
\frac{D x}{\Delta x^{2}} C_{i+1, j}^{n}-\frac{D x}{\Delta x^{2}} C_{i-1, j}^{n}+\frac{D z}{\Delta z^{2}} C_{i+1, j}^{n}- \\
\frac{D z}{\Delta z^{2}} C_{i-1, j}^{n}-\left(\frac{D x}{\Delta x^{2}}+\frac{D z}{\Delta z^{2}}\right) C_{i, j}^{n+1}-\left(\frac{D x}{\Delta x^{2}}+\frac{D z}{\Delta z^{2}}\right) C_{i, j}^{n-2}
\end{gathered}
$$

\section{Where:}

$C_{i, j}^{n}$ is the pollutant concentration at the grid node $\mathrm{i}, \mathrm{j}$ at time $\mathrm{n}$.

$u_{i, j}$ and $v_{i, j}$ are the permeability velocities in 2 directions $\mathrm{x}$ and $\mathrm{z}$ at the grid node $\mathrm{i}, \mathrm{j}$.
Convert Eq. (5) we obtain:

$$
\begin{gathered}
\left(\frac{1}{2 \Delta t}+\frac{D x}{\Delta x^{2}}+\frac{D z}{\Delta z^{2}}\right) C_{i, j}^{n+1}= \\
\left(\frac{u}{2 \Delta x}+\frac{D x}{\Delta x^{2}}\right) C_{i-1, j}^{n}+\left(\frac{v}{2 \Delta z}+\frac{D z}{\Delta z^{2}}\right) C_{i, j-1}^{n}+ \\
\left(\frac{1}{2 \Delta t}-\frac{u_{i+1, j}-u_{i-1, j}}{2 \Delta x}-\frac{v_{i, j+1}-v_{i, j-1}}{2 \Delta z}\right) C_{i, j}^{n}- \\
\left(\frac{D x}{\Delta x^{2}}+\frac{D z}{\Delta z^{2}}\right) C_{i, j}^{n-1}+\left(\frac{D x}{\Delta x^{2}}+\frac{u}{2 \Delta x}\right) C_{i+1, j}^{n}+ \\
\left(\frac{D z}{\Delta z^{2}}-\frac{v}{2 \Delta z}\right) C_{i, j+1}^{n}
\end{gathered}
$$

In short, we have:

$$
\begin{gathered}
A C_{i, j}^{n+1}=B C_{i-1, j}^{n}+C C_{i, j-1}^{n}+D C_{i, j}^{n}+ \\
E C_{i, j}^{n-1}+F C_{i+1, j}^{n}+G C_{i, j+1}^{n}
\end{gathered}
$$

Where:

$$
\begin{aligned}
& A=\frac{1}{2 \Delta t}+\frac{D x}{\Delta x^{2}}+\frac{D z}{\Delta z^{2}} \\
& B=\frac{v}{2 \Delta z}+\frac{D z}{\Delta z^{2}} \\
& C=\frac{u}{2 \Delta x}+\frac{D x}{\Delta x^{2}} \\
& D=\frac{1}{2 \Delta t}-u_{x}-v_{z} ; \\
& E=-\left(\frac{D z}{\Delta z^{2}}+\frac{D x}{\Delta x^{2}}\right) \\
& F=\frac{D z}{\Delta z^{2}}+\frac{v}{2 \Delta t} ; \\
& G=\frac{D x}{\Delta x^{2}}-\frac{u}{2 \Delta x}
\end{aligned}
$$

Solving Eq. (7) by numerical algorithms we can calculate the variation of pollutant concentration $C(x, y, z, t)$ in the $\mathrm{x}, \mathrm{z}$ directions over time $t$. In this study, the following FEM algorithm is constructed and the Matlab programming syntaxes are employed to implement the proposed algorithm.

FEM Algorithm: Calculating $C_{i, j}^{t}$ at a grid node $\mathrm{i}, \mathrm{j}$ at a time $\mathrm{n}$

Inputs: Initial concentration $C_{0}$; Parameters $D x=D y, D z$, $u=v, w$; Time step $\Delta t$; Increments of the grid $\Delta x=\Delta y, \Delta z$; Upper limitations of the increments $n_{t}, n_{x}, n_{z}$;

Output: $C_{i, j}^{t}$

Begin

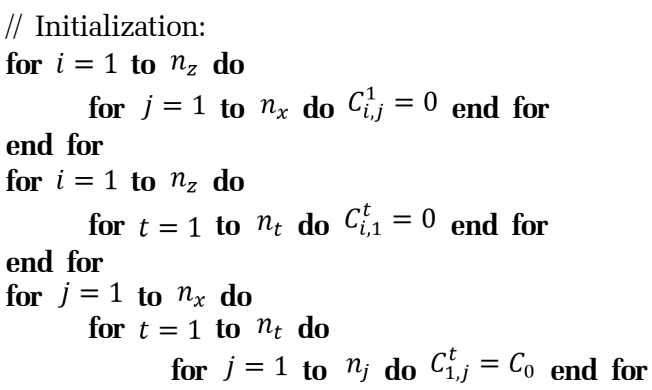

end for 


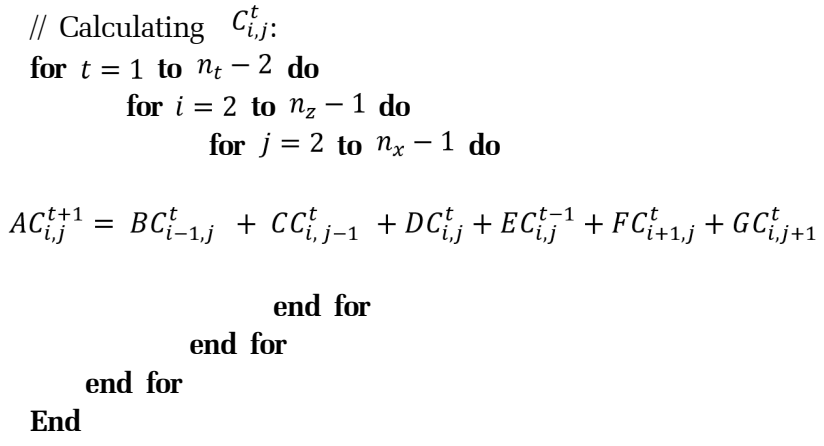

\section{Results and Discussion}

The proposed novel mathematical modelling procedure and the FME-based algorithm for simulation of the spread of pollutants in soil layers due to the leachate migration from the MSW landfill were successfully developed and demonstrated, to investigate and predict the possibility of spreading the heavy metal concentrations such as Arsenic in the soil at the nearby areas of the landfill site.

Pollutants and heavy metals in the leachate disposal from landfills discharge to soil layers, spread deep into the inner soil layers, and eventually penetrate to aquifers. As a result, this problem leads to the threat of environmental devastation, water pollution in both the short and long-term.

As presented in previous sections, the investigation of the arsenic spread in the case study of the Kieu Ky landfill was done via the following 2 main steps: (1) Determining the arsenic concentration in the nearby areas of the Kieu Ky landfill by the empirical research based on the statistical method; and (2) The measured arsenic concentration at the nearby areas of the Kieu Ky landfill is used as the initial value to implement the proposed mathematical modelling procedure and the FME-based algorithm, to study the arsenic propagation along the leachate in the ground. The soil samples for the experimental analysis in the laboratory to measure the arsenic concentration were collected from the drilling holes at different locations in the Kieu Ky landfill (Fig. 3). The concentration of arsenic heavy metal was determined through 16 soil samples in two boreholes which were drilled with the diverse depth for analysis via the use of the ICP-MS-Inductively Coupled Plasma Mass Spectrometer of high precision atomic emission spectrometry (AES). The experimental analysis showed that initial arsenic concentration is .

The detailed calculation and simulation results to demonstrate the distribution of arsenic concentration over time in the horizontal and vertical directions (Fig. 4) are presented in Fig. 5. The change of the arsenic concentration along the vertical direction, the depth $\mathrm{Z}$, and the horizontal direction $\mathrm{X}$ over time, is illustrated in detail in Fig. 6; and it is clearly shown that, the arsenic concentration changes gradually in depth and the horizontal direction over time. Generally, it can be concluded that, the contaminants spread in both the vertical and horizontal directions as time increases; and at the time constant (e.g., months 2, 4, 5, 6, 8, 10, 15 as illustrated in Fig. 6), the concentration of a contaminant gradually decreases along the three directions of the soil space (X, $\mathrm{Z}$ and $\mathrm{Z}$ directions in Fig. 4).
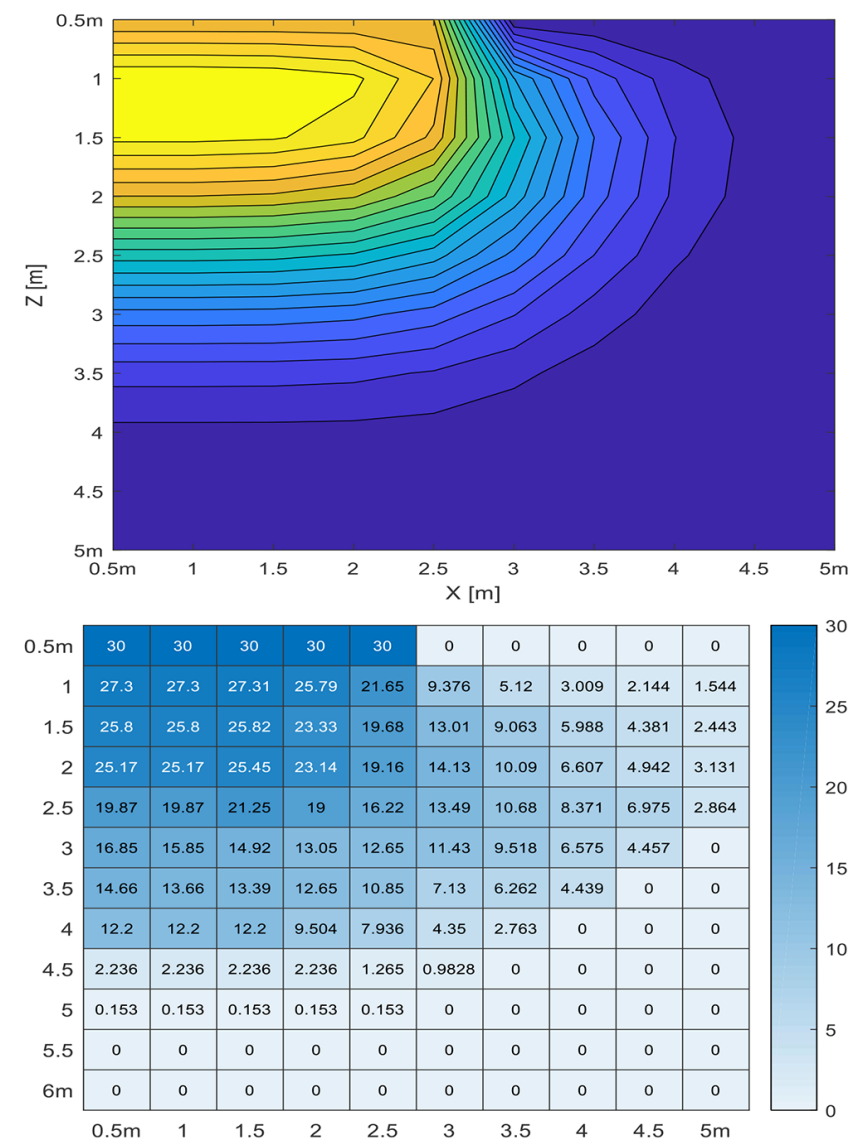

Fig. 5. Distribution of arsenic concentration in 10 months in the horizontal $\mathrm{X}$ and vertical $\mathrm{Z}$ directions.

Concretely, Fig. 6(a) presents arsenic concentration variation along the vertical direction $\mathrm{Z}$ in Months 2, 4, 6 and 8. At the distance $Z=1.5 \mathrm{~m}$ from the starting migration point of the arsenic pollutant, the arsenic concentration changes gradually over time; for example, in Month 8, the arsenic concentration is $22.4 \mathrm{mg} / \mathrm{kg}$ approximately at the depth of $3.0 \mathrm{~m}$, and the arsenic concentration value spreads in the vertical direction, it reaches the depth $\mathrm{Z}=$ $6.0 \mathrm{~m}$. Fig. 6(b) presents the change of arsenic concentration along the horizontal direction $\mathrm{X}$ over time at the depth $\mathrm{Z}=2.0 \mathrm{~m}$. The arsenic concentration spreads in the horizontal direction considerably; in Month 15, there is a significant arsenic amount at a distance $\mathrm{X}=8.0 \mathrm{~m}$ from the point in which the leachate starts spreading nominally.

In conclusions, the simulation results show that, the arsenic concentration spreads in the vertical direction at the depth $\mathrm{Z}=$ $5 \div 6 \mathrm{~m}$ from the surface after 8 months, and it spreads in the horizonal direction, at the distance $X=7 \div 8 \mathrm{~m}$ after 15 months.

The simulation results demonstrate that, the proposed mathematical modelling method is compact and applicable to simulate the distribution of the contaminant concentration over time and over distance, in the horizontal and vertical directions. By using the concepts of the concentration gradient vector $\nabla C(x, y, z, t)$, the divergence of the gradient field $(\nabla \cdot)$ and the element-wise product operator $\odot$, the advection-diffusion mechanism of a heavy metal 

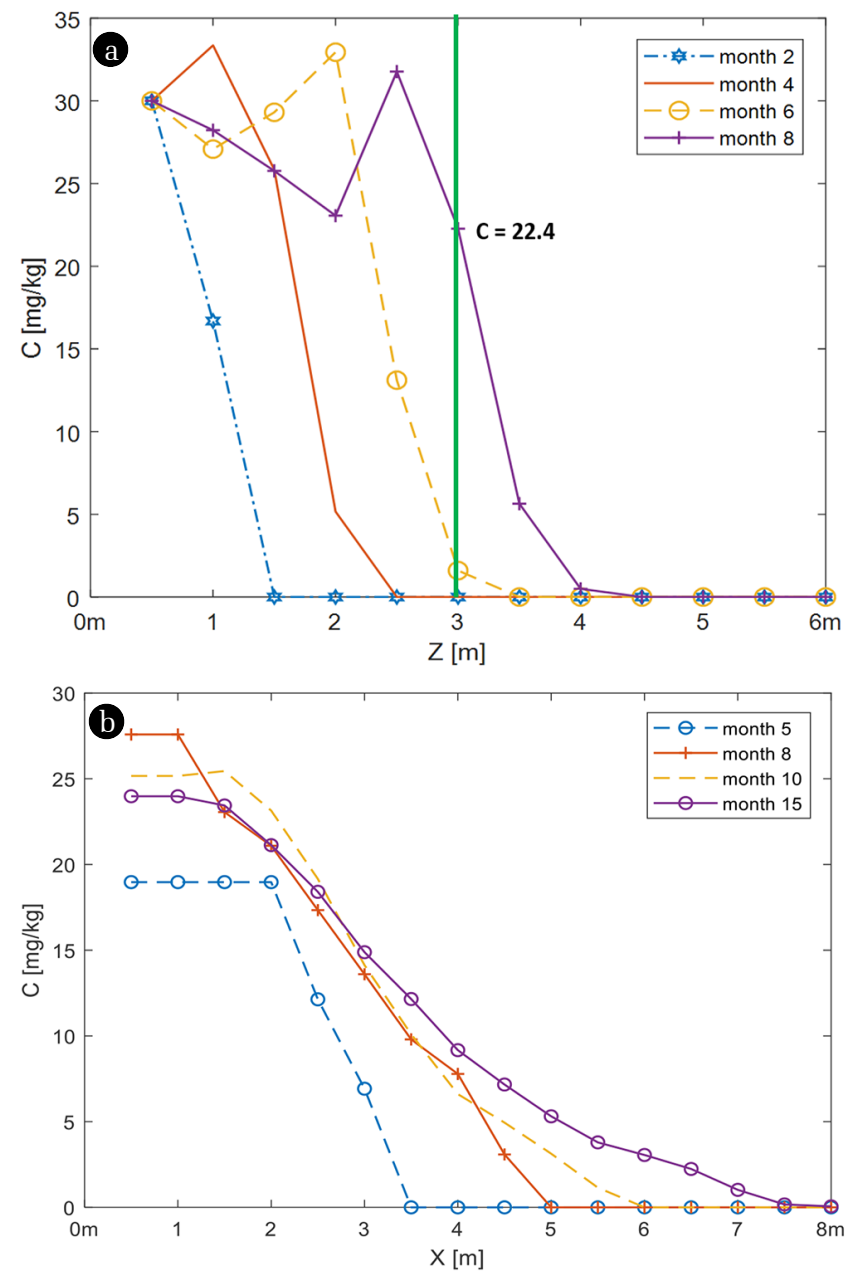

Fig. 6. (a) The arsenic concentration variation along the vertical direction, the depth Z, over time. (b) The change of arsenic concentration along the horizontal direction $\mathrm{X}$ over time at the depth $\mathrm{Z}=$ $2.0 \mathrm{~m}$.

contaminant is formulated explitly and effectively, which briefly and clearly describe the physical nature of the disperation process. In addition, the use of FEM for constructing the numerical algorithm is robust and efficient in the aspects of mathematical computation and simulation. The theoretical investigation and the implemented case study show that, the proposed mathematical modelling procedure and the FEM-based algorithms can be potentially and effectively applied to model and predict the spread of different heavy metals in the MSW landfill sites.

By using the gradient vector field approach, the derivation of the partial derivative equation which describes the pollutant concentration variation in the 3D space over time is carried out in an effective manner. In comparison with the previous works [10, $17,19,22,23]$, the derivation process of the governing equation is more explicit and generic, and it gives a comprehensive understanding about the physical nature of the spread of a contaminant in a leachate flow. In addition, when compared to the previous studies [8, 21, 23-25, 28-30], the strong points of the proposed FEM algorithm in this study can be recognized via the capability of simulating the leachate immigration in the 3D space of soil layers. By using the proposed algorithm, the time-varying of the concentration of a heavy metal in the horizontal and vertical directions can be effectively calculated and simulated.

\section{Conclusions}

The MSW landfills create the environmental pollutions due to problems related to toxins, leachate, and greenhouse gas, which are the growing environmental concerns, especially in developing countries. Many solid wastes contain toxic substances with concentrations of heavy metals, organic compounds and toxic contents; therefore, the leakage of leachate can cause serious pollutions of groundwater and natural ecosystems. In this study, a novel mathematical modelling for simulating the spread of heavy metals in solid waste landfills was presented and discussed, with the focus on simulations of the pollutant concentration variation in the leachate flow in the $3 \mathrm{D}$ space of soil in the time evolution, based on a newly developed FEM-based algorithm. By using the concepts of the concentration gradient vector $\nabla C(x, y, z, t)$, the divergence of the gradient field $(\nabla \cdot)$ and the element-wise product operator $\odot$, the advection - diffusion model of the leachate migration is formulated in a compact and simplified manner.

The newly proposed mathematical modeling and FEM-based algorithm were validated, analyzed and discussed, in which a case study was implemented, in which the soil samples collected from the actual MSW landfill and analyzed in the laboratory, to determine the arsenic concentration at the MSW landfill, and the measured arsenic concentration was used as the initial value to implement the proposed mathematical modelling procedure and the FME-based algorithm, to study the arsenic propagation along the leachate in the ground. The spread of arsenic concentration in 3D space of soil was then successfully calculated and simulated in the time evolution.

It is well-demonstrated that, the proposed mathematical modeling and FEM-based algorithm can be effectively applied to model and predict the spread of different heavy metals in the other MSW landfill sites. The quality and accuracy of the proposed mathematical modelling and FEM-based algorithm can be further enhanced, if there are more data collected from the actual MSW landfill for validations and updates, especially with the use of the latest development in heavy metal detection sensors and platforms for real-time data collection and analysis [39].

The computational and simulation results showed that there is a potential risk of the arsenic pollution in the areas around the MSW landfill; and the arsenic spread is a directly caused pollution of the underground water and soil layers in the adjacent areas of the MSW landfill site. The findings of a study can be applied to develop effective solutions and policies for solid waste classification and management to minimize the negative impacts from the potential risk of the toxic heavy metal pollutions as well as soil contamination and groundwater pollution in the areas nearby the MSW landfill, especially for the cases in which the solid wastes are disposed directly to the landfills without any classification, and for the cases of uncontrollable MSW landfill sites. 


\section{Nomenclature}

\begin{tabular}{|c|c|}
\hline MSW & Municipal solid waste \\
\hline $3 \mathrm{D}$ & Three dimensions \\
\hline FEM & Finite element method \\
\hline$C(x, y, z, t)$ & The concentration function of a contaminant \\
\hline$\nabla C$ & The gradient field of $\mathrm{C}(\mathrm{x}, \mathrm{y}, \mathrm{z}, \mathrm{t})$ \\
\hline Oxyz & The Cartesian coordinate system \\
\hline $\boldsymbol{v}=\left[\begin{array}{lll}u & v & w\end{array}\right]^{T}$ & The velocities of the desperation \\
\hline $\boldsymbol{D}=\left[\begin{array}{lll}D_{x} & D_{y} & D_{z}\end{array}\right]^{T}$ & The dispersion coefficients \\
\hline$\nabla \cdot$ & The divergence of the gradient field \\
\hline$\odot$ & $\begin{array}{l}\text { The element-wise product (the Hadamard } \\
\text { product) of two vectors }\end{array}$ \\
\hline$R$ & $\begin{array}{l}\text { The change of the concentration in the soil } \\
\text { media space due to other chemical reactions } \\
\text { and physicochemical interactions }\end{array}$ \\
\hline$C_{i, j}^{n}$ & $\begin{array}{l}\text { The pollutant concentration at the grid node } \\
\mathrm{i}, \mathrm{j} \text { at time } \mathrm{n}\end{array}$ \\
\hline$u_{i, j}$ and $v_{i, j}$ & $\begin{array}{l}\text { The permeability velocities in } 2 \text { directions } x \\
\text { and } z \text { at the grid node } i, j\end{array}$ \\
\hline$\Delta x$ and $\Delta z$ & The grid dimensions in $\mathrm{x}$ and $\mathrm{z}$ directions \\
\hline$\Delta t$ & The time step \\
\hline
\end{tabular}

\section{Author Contributions}

N.H.H. (Senior Lecturer) implemented mathematical modelling and Finite Element analysis, conducted all the experiments and wrote the manuscript. A.M.C. (Associate Professor) implemented mathematical modelling and Finite Element analysis and wrote the manuscript. K.T.T.N. (Professor) revised the manuscript. C.H.L. (Senior Lecturer) wrote and revised the manuscript.

\section{References}

1. Bag S, Mondal N, Dubey R. Modeling barriers of solid waste to energy practices: An Indian perspective. Global J. Environ. Sci. Manage. 2016;2(1):39-48.

2. Giang NV, Kochanek K, Vu NT, Duan NB. Landfill leachate assessment by hydrological and geophysical data: case study NamSon, Hanoi, Vietnam. J. Mater. Cycles Waste Manag. 2018;20:1648-1662.

3. Nima Heidarzadeh, Paria Parhizi. Improving the permeability and adsorption of phenol by organophilic clay in clay liners. Environ. Eng. Res. 2020;25(1):96-103.

4. Sabour MR, Amiri A. Polluting potential of post-Fenton products in landfill leachate treatment. Global J. Environ. Sci. Manage. 2017;3(2):177-186.

5. Ikpe Aniekan Essienubong, Ebunilo Patrick Okechukwu, Sadjere Godwin Ejuvwedia. Effects of waste dumpsites on geotechnical properties of the underlying soils in wet season. Environ. Eng. Res. 2019;24(2):289-297.

6. De S, Maiti S, Hazra T, Debsarkar A, Dutta A. Leachate characterization and identification of dominant pollutants using leachate pollution index for an uncontrolled landfill site. Global J.
Environ. Sci. Manage. 2016;2:177-186.

7. Gao G, Fu B, Zhan H, Ma Y. Contaminant transport in soil with depth-dependent reaction coefficients and time-dependent boundary conditions. Water Res. 2013;47(7):2507-2522.

8. Mirbagheri F, Samadi SA. Effects of wastewater by products on the movement of heavy metals and content of soils, crops and groundwater. In: 6th International Perspectives on Water Resources \& the Environment conference-ASCE-EWRI; 7-9 January 2013; Turkey.

9. Sharma SP, Lewis HD. Waste containment systems, waste stabilization, and landfills: design and evaluation. John Wiley \& Sons, Inc. 1994.

10. Varank G, Demir A, Top S, et al. Migration behavior of landfill leachate contaminants through alternative composite liners. Sci. Total Environ. 2011;409(17):3183-3196.

11. Benson C H, Daniel D E, Boutwell G P. Field performance of compacted clay liners. J. Geotech. Geoenviron. Eng. 1999;125(5):390-403.

12. Hoor A, Kerry Rowe R. Potential for desiccation of geosynthetic clay liners used in barrier systems. J. Geotech. Geoenviron. Eng. 2013;139(10):1648-1664.

13. Tang Q, Gu F, Zhang Y, Zhang Y, Mo J. Impact of biological clogging on the barrier performance of landfill liners. J. Environ. Manage. 2018;222:44-53.

14. Reddy K, Giri RK, Kulkarni HS. Design of vertical wells for leachate recirculation in bioreactor landfills using two-phase modeling. J. Solid Waste Technol. Manag. 2015;41(2):203-218.

15. Ogata A, Banks RB. A solution of the differential equation of longitudinal dispersion in porous media. Geological Survey Professional Paper 411-A. United States Government Printing Office, Washington; 1961. p. A1-A7.

16. Harleman DRF, Rumer RR. Longitudinal and lateral dispersion in an isotropic porous medium. J. Fluid Mech. 1963;16(3): 385-394.

17. Ogata A. Theory of dispersion in granular medium. Fluid Movement in Earth Materials. Geological survey professional paper 411-I. United States Government Printing Office, Washington; 1970. p. I1-I34.

18. Bear J. Dynamics of Fluids in Porous Media. Soil Sci. 1975;120(2):162-163.

19. Al-Niami ANS, Rushton KR. Analysis of flow against dispersion in porous media. J. Hydrol. 1977;33(1-2):87-97.

20. Abriola LM. Modeling contaminant transport in the subsurface: An interdisciplinary challenge. Rev. Geophys. 1987;25:125-134.

21. Rowe RK, Caers CJ, Barone F. Laboratory determination of diffusion and distribution coefficients of contaminants using undisturbed clayey soil. Can. Geotech. J. 1988;25(1):108-118.

22. Aral M M, Liao B. Analytical solutions for two-dimensional transport equation with time-dependent dispersion coefficients. J. Hydrol. Eng. 1996;1(1):20-32.

23. Moldrup P, Kruse CW, Yamaguchi T, Rolston DE. Modeling diffusion and reaction in soils: I. A diffusion and reaction corrected finite difference calculation scheme. Soil Sci. 1996;161(6): 347-354.

24. Ataie-Ashtiani B, Lockington DA, Volker RE. Numerical correction for finite-difference solution of the advection-dispersion equation with reaction. J. Contam. Hydrol. 1996;23(1-2):149-156. 
25. Choi J, Margetis D, Squires TM, Bazant MZ. Steady advection-diffusion around finite absorbers in two-dimensional potential flows. J. Fluid Mech. 2005;536:155-184.

26. Mirbagheri S A, Hashemi Monfared S A, Kazemi H R. Simulation modelling of pollutant transport from leachate in Shiraz landfill. Environ. Earth Sci. 2009;59(2):287-296.

27. Cianci R, Massabó M, Paladino O. An analytical solution of the advection dispersion equation in a bounded domain and its application to laboratory experiments. J. Appl. Math. 2011;2011:ID493014.

28. Shackelford C D, Daniel D E. Diffusion in saturated soil. I: Background. J. Geotech. Eng. 1991;117(3):467-484.

29. Shackelford C D \& Daniel D E. Diffusion in saturated soil. II: Results for compacted clay. J. Geotech. Eng. 1991;117(3): 485-506.

30. Lai SH, Jurinak JJ. Numerical Approximation of Cation Exchange in Miscible Displacement through Soil Columns. 1971;35(6): 894-899.

31. Patil S, Chore H. Contaminant transport through porous media:
An overview of experimental and numerical studies. Adv. Environ. Res. 2014;3:45-69.

32. Thongmoon M, McKibbin R. A comparison of some numerical methods for the advection-diffusion equation. Res. Lett. Inform. Math. Sci. 2006;10:49-62.

33. Fazelabdolabadi B, Golestan MH. Towards Bayesian Quantification of Permeability in Micro-scale Porous Structures -The Database of Micro Networks. HighTech Innov. J. 2020;1(4):148-160.

34. Buaisha M, Balku S, Özalp-Yaman S. Heavy metal removal investigation in conventional activated sludge systems. Civil Eng. J. 2020;6(3):470-477.

35. Beidokhti MZ, Naeeni STO, AbdiGhahroudi MS. Biosorption of nickel (II) from aqueous solutions onto pistachio hull waste as a low-cost biosorbent. Civil Eng. J. 2019;5(2):447-457.

36. Ferrari AGM, Carrington P, Rowley-Neale SJ, Banks CE. Recent advances in portable heavy metal electrochemical sensing platforms. Environ. Sci. Water Res. Technol. 2020;6:2676-2690. 